\title{
Silicon resistor to measure temperature during rapid thermal annealing
}

\author{
B. S. Lim, ${ }^{\text {a) }}$ E. MB, and M-A. Nicolet \\ California Institute of Technology, Pasadena, California 91125
}

M. Nathan (Natan)

Martin Marietta Laboratories, Baltimore, Maryland 21227

(Received 22 December 1986; accepted for publication 2 September 1987)

\begin{abstract}
A resistor composed of a piece of $S i$ wafer and two thin silver wires attached to it, can reliably sense the temperature during rapid thermal annealing (RTA). As constant electric current passes through the $\mathrm{Si}$ piece, the resistivity change of $\mathrm{Si}$ with temperature produces a voltage signal that can be readily calibrated and converted to an actual temperature of the samples. An accuracy better than $\pm 10^{\circ} \mathrm{C}$ is achieved between $300^{\circ}$ and $600^{\circ} \mathrm{C}$.
\end{abstract}

In recent years, there have been extensive studies in the application of RTA to silicon device technologies. ${ }^{1-3}$ The common methods to measure temperature during RTA use either an optical pyrometer or a thermocouple, both of which suffer inherent limitations for the accurate measurement of RTA temperatures. ${ }^{4}$ The application of optical pyrometers in a RTA system can be complicated and is subject to problems if all potential sources of errors are not properly considered, especially at relatively low temperatures. With thermocouples, the temperature measured is that of the thermocouple junction, which is not necessarily that of the sample of interest particulariy at very short $(10 \mathrm{~s})$ times. To securely bond the thermocouple to every sample being annealed could be laborious. ${ }^{5}$

In this work, we present an alternative method to measure the RTA temperature of smail individual Si test samples. This method has been applied successfully in our previous work. ${ }^{6}$ The method is based on the principle of the rapid resistivity change with temperature of $\$$ in in intrinsic regime. ${ }^{7}$ The resistor is composed of a piece of a commercially available $p$-type $(100\rangle \mathrm{Si}$ wafer and two thin $\mathrm{Ag}$ wires attached to it. The thin Ag wires minimize heat losses and also provide the needed flexibility to place the Si piece in the RTA unit in exactly the same manner as an actual sample in order to exactly reproduce the thermal environment of actual samples. The Ag wire is attached to each end of the Si piece by alloying it between the large Si piece and another small Si piece placed on top. To ensure good wetting, aluminum foil is folded around the wire. The Si pieces are pressed together while heat is applied by a little torch ${ }^{8}$ with a very fine tip. The completed Si resistor is drawn schematically in Fig. 1 (the Si piece is of $1.5-2.5-\Omega \mathrm{cm}$ resistivity, about $2.5 \mathrm{~cm}$ long, $0.5 \mathrm{~cm}$ wide, and the diameter of the $\mathrm{Ag}$ wires is $0.005 \mathrm{in}$.). The resistor was first calibrated in an air furnace by measuring the voltage drop across the resistor as a function of temperature, at steady-state condition for constant electric current. The resistor temperature was measured with a digital thermometer whose thermocouple was clamped to it to minimize temperature differences. The voltage reading was linear with the electric current $(I \leqslant 20 \mathrm{~mA})$. One example of the calibration curve for $I=10 \mathrm{~mA}$ is shown in Fig. 2 ; the estimated calibration error is well within $\pm 5^{\circ} \mathrm{C}$. The reproducibility is verified; the same output reading is obtained at a given temperature during the heating up and the cooling down cycle of the furnace. Comparisons with a thermocouple contained in the RTA unit (AG Associates, 410 heatpulse) have also been performed in situ at several temperatures. For long RTA cycles ( $>$ a couple of minutes), the two readings always agree within about $10^{\circ}-15^{\circ} \mathrm{C}$. This result also eliminates photogeneration of excess free carriers as the

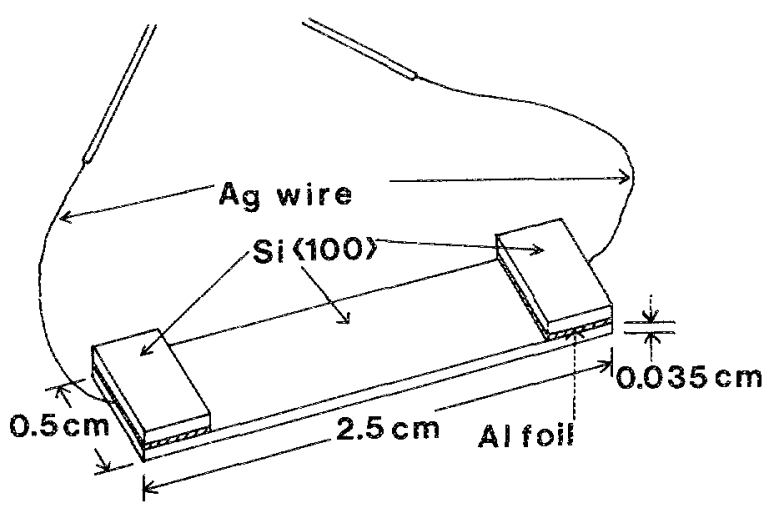

FIG. 1. Schematic drawing of Si resistor. 


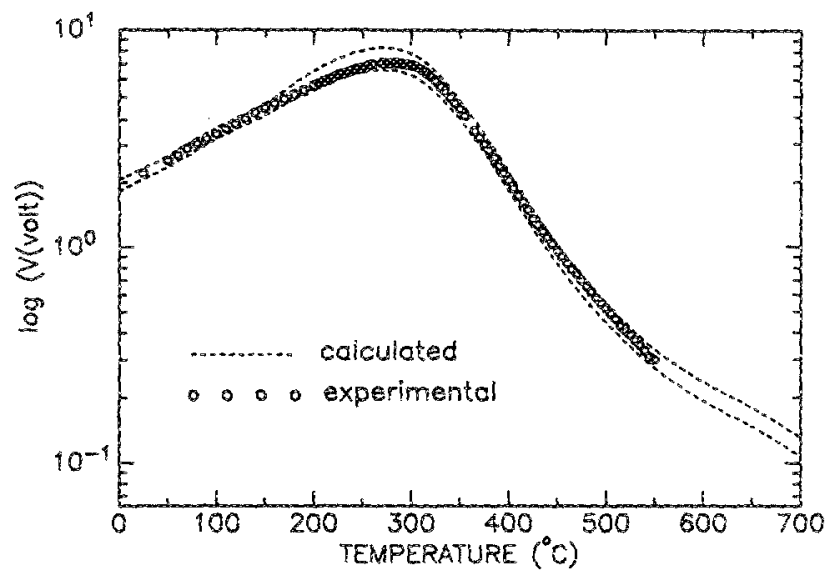

Fio. 2. Plots of voltage change vs temperature with constant current of 10 $\mathrm{mA}$. The dashed hines indicate the range of uncertainty for the theoretically predicted temperature dependence, with the room-temperature point matched to the experimental points indicated by circles.

main process responsible for the conductivity change of the resistor.

Also shown in Fig. 2 are the limits (dashed lines) calculated from the conductivity $\sigma(T)=q\left[p(T) \mu_{p}(T)\right.$ $\left.+n(T) \mu_{n}(T)\right]$, where $q$ is the electronic charge. The hole and electron concentrations $p$ and $n$ are the equilibrium values at the temperature considered; $\mu_{p}$ and $\mu_{n}$ are the hole and electron mobihities in Si. Their temperature dependence was obtained (or graphically extrapolated) from Figs. 3 and 4 of Ref. 9 . In the calculated temperature dependence, the range for the uncertainty that results from the inaccuracy in reading and estimating the numbers from the reference figures is indicated by the two dashed curves. The effective dimension of the resistor for the case of Fig, 2 is taken as 1.5 $(\mathrm{cm}) \times 0.5(\mathrm{~cm}) \times 0.035(\mathrm{~cm})$ to match the measured and the calculated points at room temperature. The experimental data are in good agreement with the predicted trend. The strong temperature dependence of the resistivity in the temperature range of $300^{\circ}$ to $600^{\circ} \mathrm{C}$ allows an accurate measurement of the temperature. The resistor is usable above $600^{\circ} \mathrm{C}$, but with decreasing accuracy.

The main advantage of the resistor over a thermocouple is in short time (10s) measurements. For short RTA runs the resistor temperature is often higher than that of a thermocouple placed in its proximity and touching the substrate (a 4-in. Si wafer) by as much as $50^{\circ} \mathrm{C}$. The resistor reading is more reliable for such siort RTAs for at least three reasons: (i) The resistor, being made of $\mathrm{Si}$, couples to the radiation field of the heat source in the same manner as a Si sample. The temperature actually measured is thus that of an object nearly identical to that of interest. (ii) The resistor has a much faster response than themocouple. In practice, the reading from the thermocouple approaches that of the resistor for long RTA runs. A pyrometer, which also has a very fast response, responds very similarly at short times to the resistor rather than to the thermocouple. (iii) A pure Alfim deposited on an oxidized Si sample exhibits morphological changes (balling up) that indicate the melting of Al when the thermocouple reads about $50^{\circ} \mathrm{C}$ less than the meiting point of $\mathrm{Al}$. The Si resistor is best suited for the temperature regime $300^{\circ}-600^{\circ} \mathrm{C}$, which is an important one for many metal-Si reactions. The simplicity, high reliability, and accuracy of this temperature sensor was an essential element in a recent experimental study of the kinetics of silicide formation by RTA. ${ }^{6}$

The authors thank $\mathbb{R}$. Gorris and $G$. Mendenila for technical assistance. Financial support from the National Science Foundation-Materials Research Group under Contract No. DMR-8421119 is gratefully acknowledged. One of the authors, B. S. Lim, would like to acknowledge the partial financial support from the Korean Science and Engineering Foundation.

\footnotetext{
a) Permanent address: Department of Mechanical Engineering, Sung K yun Kwan University, Suwon 170 , Korea.

'T. O. Sedgwick, I. Electrochem. Soc. 130, 484 (1983).

2F. M. d'Heurle, R. T. Fodgson, and C. Y. Ting, in Rapid Themal Processing, Materials Research Sociery Symposium Procedings, edited by T. 0 . Sedgwick, T. E. Seidel, and B-X. Tsaur (Materials Research Society, Pennsylvania, 1986), Vol. 52, p. 261.

${ }^{3}$ A. E. Morgan, E. K. Broadbent, M. Delino, B. Coulman, and D. K. SaGana, J. Electrochem. Soc. (to be published).

4. C. Gelpey, P. O. Siump, and J. W. Smith, in Rapid Thermal Processing, Materials Research Society Symposium Procedings, edited by T. O. Sedg wick, T. E. Seidel, and B-Y. Tsaur (Materiais Research Society, Pennsylvania, 1986), Vol. 52, p. 199.

${ }^{5}$ C. S. We, I. van der Spiegel, and I. Santiago, J. Electrochem. Soc. (to be published).

6B. \$. Lim, E. Ma, M-A. Nicolet, and M. Natan, J. Appl. Phys. 61, 5027 (1987).

'S. M. Sze, in Physics of Semiconductor Devices, 2nd ed. (Wiley, New York, 1981), Chap. 1 .

Tescom Corporation model 11-1101; tip orifice diam $0.006 \mathrm{in}$.

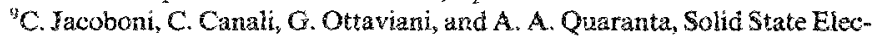
tron. $20,77(1977)$
} 